\title{
ON BLASCHKE PRODUCTS DIVERGING EVERYWHERE ON THE BOUNDARY OF THE UNIT DISC
}

\author{
C. N. LINDEN
}

Abstract. If the moduli of the zeros of a Blaschke product increase sufficiently slowly the arguments of the zeros may be so chosen that the product diverges everywhere on $\{z:|z|=1\}$.

A sequence of complex numbers $\left\{a_{n}\right\}$ in the unit disc is a Blaschke sequence if and only if $\sum_{n}\left(1-\left|a_{n}\right|\right)<\infty$. If the first $m$ terms of $\left\{a_{n}\right\}$ are zero and the remaining terms are nonzero, then the corresponding Blaschke product is defined by the formula

$$
B\left(z,\left\{a_{n}\right\}\right)=z^{m} \prod_{n>m} b\left(z, a_{n}\right)=z^{m} \prod_{n>m} \frac{\bar{a}_{n}\left(a_{n}-z\right)}{\left|a_{n}\right|\left(1-z \bar{a}_{n}\right)} .
$$

The Blaschke product converges absolutely for $|z|<1$, and, if

$$
\sum_{n}\left(1-\left|a_{n}\right|\right) \log \frac{1}{1-\left|a_{n}\right|}
$$

converges, Frostman [1] has shown that it also converges absolutely almost everywhere on $\{z:|z|=1\}$. It will be shown here that if (1) does not converge then the arguments of the terms of the sequence $\left\{a_{n}\right\}$ can be so chosen that $B\left(z,\left\{a_{n}\right\}\right)$ diverges whenever $|z|=1$. Although $I$ am not aware of the existence, in the published literature, of examples of Blaschke products which diverge everywhere on $\{z:|z|=1\}$, some unpublished examples have been obtained by Clunie and Piranian.

THEOREM. Let $\left\{r_{n}\right\}$ be a positive Blaschke sequence such that

$$
\sum_{n=1}^{\infty}\left(1-r_{n}\right) \log \left(1 /\left(1-r_{n}\right)\right)
$$

diverges, and let

$$
\theta_{N}=\sum_{n=1}^{N}\left(1-r_{n}\right) \log \frac{1}{1-r_{n}} \quad(N=1,2,3, \ldots) .
$$

Then if $a_{n}=r_{n} e^{i \theta_{n}}$ the Blaschke product $B\left(z,\left\{a_{n}\right\}\right)$ diverges for $z=e^{i \theta}(0 \leqq \theta$ $<2 \pi)$.

Received by the editors January 13, 1975.

AMS (MOS) subject classifications (1970) Primary 30A76; Secondary 30A78.

Key words and phrases. Blaschke product, divergence. 
For each natural number $k$ we choose $N(=N(k))$ to satisfy the inequality $\theta_{N-1} \leqq \theta+2 k \pi<\theta_{N}$, and, since $\theta_{n}-\theta_{n-1} \leqq 1 / e$, we can then choose $M(=M(N))$ so that

$$
\frac{1}{2}<\theta_{N}-\theta_{M}<1 .
$$

Clearly $\{N(k)\}$ and $\{M(N(k))\}$ are unbounded increasing sequences.

Elementary properties of the Blaschke factors show that

$$
b\left(e^{i \theta}, a_{n}\right)=\left(\frac{r_{n} e^{\frac{1}{2} i\left(\pi-\theta+\theta_{n}\right)}+e^{-\frac{1}{2} i\left(\pi-\theta+\theta_{n}\right)}}{\left|1-\bar{a}_{n} e^{i \theta}\right|}\right)^{2}=e^{-2 i \varphi_{n}},
$$

where

$$
\varphi_{n}=\arctan \frac{1-r_{n}}{\left(1+r_{n}\right) \tan \frac{1}{2}\left(\theta-\theta_{n}\right)} .
$$

We need to show that $\sum_{n=1}^{\infty} \varphi_{n}$ diverges.

We consider $\varphi_{n}$ on the set of integers $n$ that satisfy $M \leqq n<N$. For such $n$ we have

$$
\tan \frac{1}{2}\left(\theta-\theta_{n}\right)<\tan \frac{1}{2}\left(\theta_{N}-\theta_{n}\right)<\theta_{N}-\theta_{n}
$$

since $0<\theta_{N}-\theta_{n}<1$, whence

$$
\varphi_{n}>\arctan \frac{1-r_{n}}{2\left(\theta_{N}-\theta_{n}\right)} \text {. }
$$

If

$$
\left(1-r_{n}\right) /\left(\theta_{N}-\theta_{n}\right)>2
$$

then $\varphi_{n}>\pi / 4$. On the other hand, if (3) does not hold then

$$
\begin{aligned}
\varphi_{n} & >\frac{1-r_{n}}{4\left(\theta_{N}-\theta_{n}\right)}=\frac{\theta_{n}-\theta_{n-1}}{4 \log \left(1 /\left(1-r_{n}\right)\right)\left(\theta_{N}-\theta_{n}\right)} \\
& >\frac{1}{4 \log \left(1 /\left(1-r_{n}\right)\right)} \log \left(1+\frac{\theta_{n}-\theta_{n-1}}{\theta_{N}-\theta_{n}}\right) \\
& =\frac{\log \left(1 /\left(\theta_{N}-\theta_{n}\right)\right)-\log \left(1 /\left(\theta_{N}-\theta_{n-1}\right)\right)}{4 \log \left(1 /\left(1-r_{n}\right)\right)} \\
& \geqq \frac{\log \left(1 /\left(\theta_{N}-\theta_{n}\right)\right)}{4 \log \left(1 /\left(1-r_{n+1}\right)\right)}-\frac{\log \left(1 /\left(\theta_{N}-\theta_{n-1}\right)\right)}{4 \log \left(1 /\left(1-r_{n}\right)\right)} .
\end{aligned}
$$

Thus we have $\sum_{n=M}^{N-1} \varphi_{n}>\pi / 4$ if (3) holds for any integer $n$ in $[M, N-1]$. Otherwise we have

$$
\begin{aligned}
\sum_{n=M}^{N-1} \varphi_{n} & >\frac{\log \left(1 /\left(\theta_{N}-\theta_{N-1}\right)\right)}{4 \log \left(1 /\left(1-r_{N}\right)\right)}-\frac{\log \left(1 /\left(\theta_{N}-\theta_{M-1}\right)\right)}{4 \log \left(1 /\left(1-r_{M}\right)\right)} \\
& >\frac{1}{4}-\frac{\log \log \left(1 /\left(1-r_{N}\right)\right)}{4 \log \left(1 /\left(1-r_{N}\right)\right)}-\frac{\log 2}{4 \log \left(1 /\left(1-r_{M}\right)\right)}
\end{aligned}
$$


since $\theta_{N}-\theta_{M-1}>\frac{1}{2}$ by (2). Since $\lim _{n \rightarrow \infty} r_{n}=1$, it follows that $\sum_{n=1}^{\infty} \varphi_{n}$ diverges.

\section{REFERENCE}

1. O. Frostman, Sur les produits de Blaschke, Kungl. Fysiografiska Sällskapets i Lund Förhandlingar 12 (1942), no. 15, 169-182. MR 6, 262.

University College of Swansea, Singleton Park, Swansea, Wales, United Kingdom 